\title{
Table ronde sur la traduction des contemporains de Shakespeare
}

Jean-Michel Déprats

\section{(2) OpenEdition}

1 Journals

\section{Édition électronique}

URL : http://journals.openedition.org/shakespeare/1346

DOI : 10.4000/shakespeare.1346

ISSN : 2271-6424

Éditeur

Société Française Shakespeare

Édition imprimée

Date de publication : 1 novembre 2002

Pagination : 221-235

Référence électronique

Jean-Michel Déprats, "Table ronde sur la traduction des contemporains de Shakespeare », Actes des congrès de la Société française Shakespeare [En ligne], 20 | 2002, mis en ligne le 01 novembre 2007, consulté le 23 avril 2019. URL : http://journals.openedition.org/shakespeare/1346 ; DOI : 10.4000/ shakespeare.1346 


\section{S H A K E S P E A R E \& S E S \\ C O N T E M P O R A I N S}

Société Française Shakespeare

Actes du Congrès de 2002

$* * *$

Textes réunis et présentés par

Patricia DORVAL

publiés sous la direction de

Jean-Marie MAGUIN 


\section{TABLE RONDE SUR LA TRADUCTION \\ DES CONTEMPORAINS DE S H A K E S P E A RE}

Jean-Michel DÉPRATS. Dans quelques mois paraîtront deux volumes de la Pléiade dirigés par François Laroque et Jean-Marie Maguin et consacrés au thêatre de la Renaissance anglaise. Plus de vingt auteurs traduits, présentés et commentés par plus de vingt collaborateurs. Dans le cadre de notre colloque, et du thème de cette table ronde, il ne peut donc être question que d'évoquer quelques aspects, quelques problèmes spécifiques de traduction d'un nombre limité d'auteurs et d'œuvres (Dekker, Ford, Jonson, Kyd, Marlowe, Massinger). C'est à cette fin que nous avons réuni cinq collaborateurs de cette entreprise : Jean-Marie Maguin, Robert Ellrodt, Yves Peyré, Jean-Pierre Villquin et Michèle Willems qui parleront dans cet ordre.

Puisque une précédente table ronde intitulée «Traduire Shakespeare aujourd'hui» et animée par Jean-Pierre Maquerlot s'était concentrée sur les problèmes du vers, de l'historicité et de la théâtralité de la langue de traduction ', je propose que nous parlions plutôt des difficultés singulières auxquelles vous avez été confrontés et que nous procédions en deux temps, nous intéressant d'abord aux problèmes rencontrés dans la tragédie (élisabéthaine, jacobéenne, caroléenne) pour nous consacrer ensuite à l'examen des problèmes de traduction de la comédie citadine. Jean-Marie 
Maguin, vous êtes avec François Laroque le maître d'œuvre de ces deux volumes et vous avez traduit pour le volume I La Tragédie espagnole de Thomas Kyd. Quelles remarques souhaitez-vous faire?

Jean-Marie MAGUIN. En ce qui concerne la coordination des deux volumes de la Pléiade consacrés aux prédécesseurs, aux contemporains et aux successeurs de Shakespeare, il a été jugé souhaitable de définir certains paramètres à prendre en compte pour une collection de trente pièces étalées du début du XVIe (Anon., Everyman) à la fermeture des théâtres en 1642 (Shirley, The Cardinal), soit près d'un siècle et demi :

1- Respecter dans le style de chaque auteur : a) ce qui l'insère dans la chronologie; b) ce qui le pose par rapport au type de spectateur pour lequel il écrit (théâtre public ou privé, spectacle aristocratique). Bien que proches dans le temps, Kyd et Lyly, par exemple, écrivent pour des types de spectateur complètement différents.

2- Définir positivement ou négativement des impératifs qui donneront à la collection une unité d'attitude (c'est cette «philosophie» qui la datera) : différencier le vers de la prose, respecter (relativement parlant) le volume des différents vers dans la traduction qu'on en donne. Ne jamais excéder quatorze syllabes pour rendre un décasyllabe anglais. Éviter de reporter des éléments sémantiques d'un vers sur l'autre. Respecter strictement la linéation, comme si l'on devait pouvoir confronter le texte traduit à l'original en vis-à-vis. Respecter le profil que donne au vers la distribution des syllabes entre les pauses. Respecter les données prosodiques et rhétoriques : rimes, métaphore ou similé, allitération, anaphore, épiphore, les parallélismes de construction, en général. Proscrire le recours systématique à l'alexandrin classique. Celui-ci peut être utile, en revanche, pour traduire les distiques rimés servant à la ponctuation dramatique ou à l'énonciation gnomique. Produire un texte clair et sans «casse-mâchoire», c'est-à-dire un texte qu'un comédien peut mettre en bouche sans retouche motivée par des raisons de diction. Proscrire l'archaïsme systématique, les inversions de l'ordre normal des mots (anastrophes) non justifiées par le texte.

J'emprunterai quelques exemples pratiques au texte de La Tragédie espagnole de Thomas Kyd que j'ai traduite pour la collection. Il faut de façon générale faire face au problème de la grandiloquence qui caractérise le style de Kyd et qui est différente de la boursouflure, ridicule sur scène. Un dosage stylistique précis s'impose. 
Dans les quatres premiers vers de la pièce, il faut rendre la chute, l'effet de «bathos» du quatrième vers (un comédien peut en tirer parti). Avant de produire son texte, le traducteur doit régler mentalement le problème de l'aspect du locuteur (costume, maquillage; il s'agit d'un fantôme) :

Du temps où l'éternelle substance de mon âme

Vivait emprisonnée dans mon folâtre corps,

Chaque partie veillant aux besoins de l'autre,

J'étais un courtisan à la cour d'Espagne. (I.1.1-4)

Le choix de la traduction de «wanton», dans l'original, par «folâtre» est évidemment délibéré. "Wanton», dans la bouche de l'être mort qui s'exprime, emblématise au travers d'un maniérisme l'énergie sexuelle du vivant qu'il n'est plus. Dans Le Chevalier de l'Ardent Pilon, Beaumont fera des gorges chaudes de ce début, en accentuant l'effet de «bathos». Il faudra que le lecteur puisse retrouver cet écho dans la série des pièces. Les données intertextuelles doivent être soigneusement prises en compte d'une traduction à l'autre, ce qui n'est pas toujours facile quand on a affaire à une équipe de traducteurs différents. J'ai repris «folâtrer» pour traduire «frolic»: «King. But now Knight Marshal frolic with thy king» (I.2.96) : «Mais pour lors, Maréchal, folâtre comme ton roi». C'est un faux sens délibéré que de traduire «with» par «comme». Il est destiné à éviter une ambiguité pour le spectateur moderne. Il n'y a pas dans la pièce de suggestion d'une quelconque relation homosexuelle.

Voici maintenant un exemple de difficulté de traduction due aux rapports existants entre les données sémantiques et leurs correspondances phonétiques, très différentes d'une langue à l'autre. Il s'agit de rendre : «O eyes, no eyes, but fountain fraught with tears» (III.2.1). Quels sont les impératifs du traducteur ici ? Respecter la correctio qui structure aussi les deux vers suivants, respecter l'allitération «fountain fraught». Ne pas faire trop long. Or le vers anglais est presque uniquement fait de monosyllabes. «Pauvres yeux, non yeux» ne fonctionne pas. «Pauvres yeux, yeux non pas» est un peu moins mauvais. Mais la répétition en français est obligatoirement handicapée par la nécessité de la liaison à la première occurrence : «zyeux». L'allitération «fountains fraught» est reportée (et délayée) sur «mệlées de larmes»...

Jean-Michel DÉPRATS. Robert Ellrodt, votre talent de traducteur de poésie est connu depuis longtemps et vous avez donné au cours de la 
décennie écoulée un magnifique John Donne et un non moins impressionnant Keats aux éditions de l'Imprimerie Nationale. Quelle a été votre contribution aux volumes de la Pléiade?

Robert ELLRODT. J'ai traduit Doctor Faustus et 'Tis Pity She's a Whore. Comme Antoine Berman, je pense que pour le traducteur «il n'y a que des solutions particulières». Le vers de Marlowe, «the mighty line», dont la puissance a surpris les contemporains, m'a paru appeler souvent l'alexandrin pour marquer que la parole se hausse au dessus du ton ordinaire. Un alexandrin libéré, certes, des contraintes de la prosodie classique pour mieux suivre les détours de la méditation faustienne comme les sursauts de l'émotion tragique. Mais de Marlowe à Ford, en passant par Shakespeare, le vers blanc s'est assoupli au point de ne plus se distinguer de la prose que par une moindre imprévisibilité du rythme. Il convient alors de recourir à un vers libre proche du langage parlé, mais encore hanté par l'alexandrin dans les élans lyriques.

Une traduction peut épouser les rythmes du texte original, mais il est plus difficile de transposer les autres effets sonores: allitérations, assonances, et ce que Berman appelle la «corporéité iconique du mot» ( $L a$ traduction et la lettre ou l'auberge du lointain, Paris, rééd. 1999, p. 58-9). Il faut se résigner à cette «défectivité inhérente à l'acte traductif» (Pour une critique des traductions de John Donne, Paris, 1995, p. 86). Entendons-nous : cela ne signifie pas que le texte traduit est toujours inférieur à l'original : l'inverse peut même parfois être vrai. Mais le passage d'une langue à l'autre conduit à renoncer à certaines connotations, plus ou moins heureusement remplacées par d'autres.

La polysémie de l'expression peut rendre la tâche particulièrement ardue, notamment dans les textes shakespeariens. Cette polysémie est beaucoup moins marquée dans le style de Marlowe et même dans le style de Ford, mais on est amené à s'interroger sur le niveau de culture que l'on peut attendre du lecteur et de l'auditeur. Dans l'apostrophe célèbre de Faust à Hélène, j'ai traduit «the topless towers of Ilium» par «les tours altières d'Ilion»; mais, dans le cas d'une traduction «faite en pensant au théâtre», François Laroque et Jean-Pierre Villquin (Flammarion, 1997) ont préféré parler des «tours altières de la ville de Troie»; sans doute à juste titre. Dans 'Tis Pity She's a Whore, quand Giovanni, sur le point de posséder Annabella, s'écrie: «I would not change this minute for Elysium» (1.2.260), Jean-Michel Déprats, traduisant la pièce pour la collection Folio/Théâtre, a raison de substituer le «Paradis» à «l'Élysée» 
(que certains spectateurs prendraient peut-être pour le palais présidentiel !). Mais il m'a semblé, à tort ou a raison, que les lourds volumes de la Pléiade ne seraient pas utilisés par les acteurs pour apprendre leur rôle et que le metteur en scène pourrait, s'il le jugeait bon, adapter par endroits le «script» au public attendu. Je me suis donc senti libre de respecter les préférences de dramaturges qui présentent des héros pénétrés comme eux de culture classique; de poètes attentifs au charme des sonorités comme aux rapprochements suggérés par les termes choisis. Ainsi le lecteur pourra-t-il discerner dans les paroles de Giovanni une ironie qui, lors d'une représentation, échappe, j'en conviens, même à l'auditeur averti : ce «libertin» (ainsi dépeint par Gisèle Venet) commet à ce moment-là une erreur tragique, car il en viendra à dire «A life of pleasure is Elysium» (V.3) sans se douter que, comme Faust, il «confond l'Enfer et.l'Élysée» (Doctor Faustus, I.3).

Le traducteur de Ford ne bute pas, comme le traducteur de Shakespeare, sur des jeux de mots parfois intransposables, mais il doit demeurer attentif à des sens multiples. Quand Annabella, poignardée par Giovanni, l'appelle «brother unkind, unkind», elle s'adresse à un frère incestueux à la fois «dénaturé» et «cruel»; cependant, en choisissant une expression négative, «sans bonté», elle me semble vouloir, dans son amour intact, atténuer le reproche. Comment suggérer ces nuances diverses?

Et comment traduire le titre même de la pièce, que Henri Suhamy, cité par Gisèle Venet (p. 269), a déclaré «intraduisible»? Dommage que ce soit une putain est aujourd'hui la traduction consacrée par l'usage. Mais Ford, qui s'est souvent inspiré d'Othello, se souvenait peut-être que le Maure, voyant en Desdémone «a whore» et décidé à la tuer, soupirait : «but yet the pity of it, Iago !» (IV.1.194-5). La «pitié» est une émotion, «dommage» une sèche constatation. Le Cardinal, dira-t-on, est peu susceptible d'éprouver de la pitié. Soit ! Mais sa remarque ne serait-elle pas précisément reprise dans le titre pour nous conduire à une autre interprétation du destin d'Annabella et pour appeler à une authentique pitié ? Quant à «putain», le terme français est inexact - l'hérö̈ne n'est assurément pas une "prostituée» - et le mot tombe dans le registre de la vulgarité. Ce n'est pas le cas de «whore», car on lui substitue aujourd'hui d'autres termes dans la langue parlée. Qui plus est, au dix-septième siècle, sa présence dans la Bible pouvait l'élever à un autre registre. Il est d'ailleurs ironique que ce jugement soit prononcé par le Cardinal, représentant de l'Église de Rome, identifiée par les Protestants à «the great whore», la Babylone de l'Apocalypse (xvii). On ne peut recréer ces échos, 
mais ne devrait-on pas éviter les résonances inopportunes ? Maeterlinck a tourné la difficulté en donnant à son «adaptation» le nom de l'héroïne : on ne peut le faire dans une traduction. J'avais envisagé de rendre ainsi les deux derniers vers rimés :

D'une femme si jeune, si riche en dons de la Nature, Qui ne dirait : C'est pitié qu'elle soit impure.

«Impure», il est vrai, a d'autres connotations que «whore», mais sont-elles injustifiables? L'inceste porte la plus grave atteinte à ce désir spontané qu'éprouve l'homme de garder pure l'image de la mère et de la sœur. Un metteur en scène aura-t-il un jour l'audace de changer le titre ? Non par pudeur : en 1960 Georges Pillement (ou son éditeur) l'écrivait encore Dommage qu'elle soit une $P$... ; ce qui aggravait la vulgarité. Mais par fidélité à l'esprit même d'une œuvre audacieuse et intense, et à ce style pur, ce vers blanc au «rythme distinct et personnel», dont T.S. Eliot a reconnu la «beauté particulière».

Jean-Michel DÉPRATS. Yves Peyré, vous avez rencontré, je crois, des difficultés très spécifiques sur des cuvres qui n'avaient jamais été traduites en français auparavant. Comment résumeriez-vous votre expérience?

Yves PEYRÉ. Je me contenterai d'évoquer très brièvement quelques questions qui se sont posées à moi lors de la traduction de deux pièces de contemporains de Shakespeare, Patient Grissil de Dekker, Chettle et Haughton (1600), publiée dans L'Histoire de Griselda (Presses Universitaires du Mirail, 2002) et The Roman Actor, de Philip Massinger (1626), à paraître dans le second volume des Contemporains de Shakespeare en préparation pour la collection de la Pléiade.

Le texte de Patient Grissil est d'une extraordinaire variété stylistique. Il convient de respecter les différents registres dans le texte français, en trouvant des variations équivalentes à celles qui existent entre le pentamètre non rimé, le pentamètre rimé, la prosodie des chansons, et divers types de prose, allant du langage précieux au langage populaire du bouffon. La préciosité pédante est ici surtout une question de vocabulaire («my collocution tendeth to Sir Owen's magnifying», «ma collocution tend à la magnification de sir Owen»). Certains néologismes du texte anglais ne sont plus clairement compréhensibles aujourd'hui : le traducteur 
a fait le choix d'inventer une signification compatible avec le contexte ; c'est ainsi que «imprision» est devenu «malencontre». La prose du bouffon abonde en jeux de mots qui ont aisément, et presque naturellement, trouvé un équivalent français : «Unless I ride post, I kiss the post», «Si je ne me barre pas vite, je vais tout trouver barré»; «They cannot choose but ride well, because every good wit rides them», «Il faut qu'ils montent bien, parce que tout homme d'esprit les démonte aisément» ; «There's no meat toward ; the lady is untoward», «La table n'est pas dressée ? C'est la maîtresse de maison qui a besoin d'être dressée».

Le problème le plus épineux est celui de l'accent gallois de sir Owen et de son épouse Gwenthyan. Certains jeux de mots passent facilement : «is prave, tis repatoes, I patoes money out a cry», «il est superbe ce rabat, et mes dépenses, il ne les rabat pas». Mais qu'est-ce qu'un accent gallois en français ? Faute de pouvoir reproduire de façon convaincante et compréhensible ce que serait le français d'un Gallois, dans un premier temps, on a essayé de fournir un équivalent des distorsions phonétiques et des irrégularités syntaxiques; le texte français en devenait alors très difficile à comprendre et l'on risquait de perdre toute efficacité dramatique. Il n'a pas semblé envisageable non plus de substituer à «l'accent gallois» un accent régional repérable, alsacien, auvergnat, ou même breton, qui introduirait une contradiction avec la fiction mise en scène. Ponctuellement, on a fait le choix de ne pas «traduire» l'accent, sauf en le symbolisant, sur la page, par une seule variation phonétique qui n'a d'autre fonction que de signaler à l'acteur que la mise en scène devra prendre en charge un élément dont n'a pas su rendre compte le traducteur.

Pas plus que Patient Grissil, The Roman Actor n'avait été traduit en français, à l'exception de deux courts passages traduits en prose par Joseph de Smet et publiés dans les Cahiers $d u$ Sud en 1933 et en 1940. La traduction actuelle utilise un vers souple variant entre 8 et 14 syllabes. La pièce possède une grande unité de style et de ton, avec une prédominance du style oratoire qui se traduit par de très longues tirades, dont les phrases peuvent s'étendre sur 8 à 10 vers. L'essentiel du travail de traduction est ici de produire un texte qui conserve le souffle de l'original. Il est donc exclu de segmenter la syntaxe, dont il convient de conserver l'ampleur. Mais cela n'est possible qu'en accordant une attention minutieuse à l'articulation entre l'agencement d'ensemble et la structuration du détail. Pour cette raison, on a privilégié, à l'intérieur de chaque phrase, des unités syntaxiques de quatre, six ou huit syllabes; on a ménagé des points 
d'ancrage pour la voix, et étudié l'alternance des pauses, soit en fin de vers, soit au milieu du vers.

Jean-Michel DÉPRATS. Jean-Pierre Villquin, avec Jean-Marie Maguin, qui a traduit un Roi Lear non encore publié, vous partagez l'expérience d'un premier travail de traducteur de Shakespeare (Roméo et Juliette) qui suivait une traduction de Faust jouée, comme Roméo et Juliette, par une troupe de Nantes. Quelles pièces avez-vous traduites pour la Pléiade et quelles sont vos analyses ?

Jean-Pierre VILLQUIN. Les textes auxquels je suis confronté couvrent un peu plus d'un siècle depuis 1534 jusqu'aux années 1640. Il s'agit surtout de pièces de théâtre, mais les mêmes questions se posent quel que soit le genre, quels que soient les auteurs et quelle que soit la date à laquelle les textes ont été écrits ou publiés. On peut aborder les problèmes sous trois angles : la compréhension, la traduction et ce qu'on peut appeler la théâtralité du texte. Je ferai allusion ici à deux pièces : l'une de Ben Jonson, Bartholomew Fair (jouée en 1614) (édition utilisée : E.A. Horsman, ed., dans The Revels Plays, Methuen, 1960, rep. 1965) et l'autre écrite en collaboration par Ben Jonson, Chapman et Marston, Eastward Ho! (imprimée en 1605) (édition utilisée : Eastward Ho ! by Chapman, Jonson and Marston, ed. R.W. Van Fossen, The Revels Plays, Manchester University Press, The Johns Hopkins University Press, Baltimore, Maryland, Alden Press, Oxford, 1979).

La compréhension du texte est une évidente nécessité et la première mission du traducteur est d'être fidèle au message de l'auteur. Cette évidence se heurte aux difficultés inhérentes à une langue parfois archaïque tant sur le plan syntaxique que sur le plan lexical. L'anglais de la Renaissance est une langue vivante, évolutive, diverse, ondoyante. Chaque auteur a sa marque propre, souvent reconnaissable dans les nombreuses pièces écrites en collaboration. L'alternance de prose et de vers ne facilite pas toujours la cohérence et la fluidité du texte. Les allusions historiques, anecdotiques et topiques chargent le sens et multiplient des perspectives qui aujourd'hui nous échappent. Les genres (narratifs, épiques, lyriques, comiques, tragiques) se côtoient, se chevauchent, se répondent et en outre se reflètent souvent dans le miroir déformant de la parodie.

Shakespeare n'est pas le seul auteur à «corrompre les mots». Avec les «double-entendre», les doubles ou triples sens, sous-entendus, pataquès 
et «noms étiquettes» nous atteignons les frontières de l'intraduisible comme dans les dialogues d'ivrognes que l'on trouve dans Eastward Ho! Cet excès, cette ivresse des mots, paradoxalement, libère parfois le traducteur. Les notes si abondantes dans les éditions modernes des textes de la Renaissance révèlent les nuances, les diverses interprétations, les contradictions parfois et tout simplement la difficulté de compréhension. Les notes qui déjà rompent le fil de la lecture sont impossibles au théâtre, on ne peut pas expliquer un calembour. Il faut donc faire un choix souvent réducteur, rarement satisfaisant et toujours source de frustration.

Le troisième volet est la cible visée par la traduction, c'est-à-dire l'usage qui en est fait. Le texte de théâtre est conçu pour être joué, pour être dit. Il y a donc un triple problème, le texte d'arrivée doit être fidèle, pouvoir être dit et rester concis. Ces problèmes sont encore plus sensibles quand il s'agit de vers. Faut-il se contenter d'aller à la ligne dans une traduction imprimée, faut-il se risquer à substituer de mauvais et souvent de faux alexandrins aux traditionnels pentamètres iambiques, faut-il s'attacher au rythme, essayer de respecter les accents ou tout simplement et plus modestement écouter les mots pour qu'un acteur puisse en restituer par la voix les sens, les couleurs et les sonorités? La mission semble impossible quand on pense à la difficulté de traduire de simples monosyllabes comme «now» et quand on pense à la difficulté de respecter la cohérence de longues phrases ou de métaphores complexes. Comment tenir compte en français d'une ponctuation anglaise qui coordonne les phrases et rythme si parfaitement la respiration de l'acteur ? L'espoir du traducteur est que l'acteur puisse s'approprier le texte pour en faire, sur scène, une matière vivante.

Pour illustrer ces quelques remarques, je prendrai deux exemples qui montrent, à mes yeux, la difficulté de la compréhension et de la traduction. Le premier est extrait de Bartholomew Fair (III.4.65-8). La Guêpe (Wasp), l'intendant et serviteur de Coquebin (Cokes), est chargé comme un baudet des babioles que son maître a achetées à la foire et il répond à Chamaillard (Quarlous) qui lui demande s'il déménage : «A pretty question! ... you may know whose beast I am by the burden. If the pannier-man's jack were ever better known by his loins of mutton, I'll be flay'd and feed dogs for him, when his time comes». Quel est donc ce «the pannier-man's jack» et doit-on prendre «loins of mutton» au premier degré ou comme le dit pudiquement Partridge dans son Shakespeare's Bawdy dans le sens de «woman regarded as food for the satisfaction of man's sexual appetite»? 
Ici comme ailleurs je laisse à la sagacité et à l'érudition des lecteurs le soin de trouver la solution.

Le deuxième exemple est tiré de Eastward Ho! (II.2.313-21). La complexité de la métaphore qui suit, même si elle est compréhensible, ne suscitera sans doute que peu d'échos sur une scène contemporaine. Il y a bien longtemps qu'on a oublié qu'un chien dans une roue pouvait activer une rôtissoire. Vifargent (Quicksilver), apprenti bijoutier peu scrupuleux brosse un tableau peu flatteur du mariage et de la fille de son patron, future épouse de Messire Tromblon du Clinquant (Sir Petronel Flash) : «... and how she will tie you to your tackling till she be with child, a dog would not endure. Nay, there is no turnspit dog bound to his wheel more servilely than you shall be to her wheel ; for as that dog can never climb the top of his wheel but when the top comes under him, so shall you never climb the top of her contentment but when she is under you».

Jonson semble se délecter du «nom étiquette» qui est un extraordinaire raccourci du personnage. Parfois l'équivalent se trouve dans le théâtre français du temps, mais souvent il faut trouver ùn substitut dont la pertinence n'est pas toujours évidente. C'est ainsi que dans Eastward Ho! Touchstone, le bijoutier devient Touchaud, Slitgut, l'apprenti boucher devient Fendlapanse, Holdfast, le geôlier, Doubletour, Sindefy, pécheresse revendiquant sa condition et maîtresse de Vifargent, sera Pousseauvice. Dans Bartholomew Fair, Littlewit, le procureur devient Nigaudin, Winwife, amateur de riches partis, devient Courtladot, Overdo, le zélé juge de paix, Tropenfait et Val Cutting, petit malfrat bagarreur, Valentin le Désosseur. Quant à Zeal-of-the-Land Busy, l'homme de Banbury, puritain bigot et hypocrite, il devient Lagité, de son prénom Zèle-de-la-Terre. Jonson raille ainsi l'habitude qu'avaient les puritains de donner des prénoms de ce genre à leurs enfants.

Étrange paradoxe que le travail du traducteur qui efface un texte pour lui substituer une image la moins déformée possible dans le miroir d'une autre langue. L'expérience prouve qu'entre la théorie et la réalité de la traduction il y a souvent un fossé difficilement franchissable. La fidélité à des textes datant de plus de trois cents ans n'est pas nécessairement contradictoire avec une certaine modernité. N'oublions jamais que le texte traduit est fait pour nos contemporains et, quand il s'agit de thêatre, pour être joué. L'écrit original reste figé, immuable, impénétrable pour qui ne connaît pas la langue, la traduction en donne un reflet éphémère que seul l'acteur peut rendre vivant. 
Jean-Michel DÉPRATS. Michèle Willems, tout votre effort a porté sur Ben Jonson et sur ses comédies les plus connues, Volpone et L'Alchimiste, qui recèlent l'une et l'autre des difficultés très considérables. Quelles sont-elles et quelles sont vos suggestions et vos propositions?

Michèle WILLEMS. Ben Jonson, contrairement à Shakespeare n'a guère suscité la traduction; il existe certes quelques rares traductions de type universitaire, le plus souvent incomplètes, mais les textes utilisés sur les scènes françaises sont généralement des adaptations (le Volpone de Jules Romains, ou L'Alchimiste de Marcel Moussy). La question de la retraduction ne se pose donc pas : au contraire, le traducteur se trouve seul face à certains passages incompréhensibles pour lesquels les éditions anglaises ne sont pas d'un grand secours.

Mais, contrairement encore au traducteur de Shakespeare, il ne rencontre guère de problèmes de texte, puisque Ben Jonson a lui-même supervisé le Folio de 1616 ; cet avantage est toutefois à double tranchant : en effet, alors que le texte shakespearien s'adresse sans conteste au spectateur, le dilemme sur la nature du destinataire (et donc sur la nature du projet) est inscrit dans la pratique jonsonienne et il se trouve accentué par le passage des siècles et par le changement de langue. Ben Jonson s'adresse-t-il au lecteur ou au spectateur? Les prologues semblent viser prioritairement le lecteur. Les acrostiches, qui résument l'argument de nos deux pièces, doivent fonctionner pour l'œil aussi bien que pour l'oreille (il sont écrits en pentamètres rimés et la traduction en alexandrins semble s'imposer). Celui qui ouvre L'Alchimiste offre un exemple de quasiintraduisibilité puisqu'en français le titre de la pièce comporte une apostrophe qui ne peut figurer en début de vers. Ma traduction propose donc de faire passer l'Argument de 12 à 11 vers en gérant au mieux tous les autres paramètres (sens, rythme, rimes, etc.).

Le texte de Ben Jonson est caractérisé par une abondance verbale qui n'égale sans doute pas la prolixité shakespearienne, et par un recours plus systématique aux allusions savantes. Par exemple, dans le divertissement offert à Volpone par ses monstres (I.2.6-17), Nano reprend l'histoire de la transmigration de l'âme de Pythagore racontée par Lucien dans Le dialogue du savetier et du coq .

Ce qui est spécifique à Ben Jonson, en particulier dans L'Alchimiste, c'est la multiplicité des jargons, comme lorsque Surly, dans une longue tirade satirique, passe en revue tous les termes employés par les alchimistes pour abuser les esprits crédules (II.3.183-97); mais, 
contrairement à ce qu'on pense parfois, le plus difficile, ce n'est pas de traduire la langue de l'alchimie : la pièce empile une variété de jargons qui tous relèvent de la fausse science et visent à berner les nigauds en les impressionnant. Dans ces cas-là, il faut surtout que la traduction jargonne, elle aussi. Mais comme le texte de Jonson offre une moindre richesse métaphorique que celui de Shakespeare, il impose rarement au traducteur de choisir entre diverses significations. En fait, dans la traduction d'une comédie de Jonson, la déperdition essentielle n'est ni sémantique ni poétique, mais culturelle. La comédie citadine (même lorsqu'elle est, comme Volpone, située à Venise) est difficilement dissociable du contexte jacobéen, comme lorsque Doll Common rappelle à ses comparses le danger que représentent leurs «precise neighbours» qui

Would run themselves from breath, to see me ride,
Or you t' have but a hole to thrust your heads in,
For which you should pay ear-rent ? No, agree.
And may don Provost ride a feasting long,
In his old velvet jerkin and stain'd scarfs...

(The Alchimist, I.1.163 ; 166-70)

La traduction doit ici s'efforcer de restituer la couleur locale et de clarifier les allusions. Pour rendre le texte intelligible, elle doit aussi lui annexer de nombreuses notes explicatives, comme lorsqu'il est question des pratiques frauduleuses que dénonçaient les pamphlets contemporains. Cette inscription des pièces dans le contexte jacobéen explique sans doute l'échec des représentations en costume moderne, même en Angleterre. De la même manière, le rajeunissement opéré par la traduction shakespearienne est ici beaucoup plus aléatoire, car le texte est moins «ouvert», donc moins modernisable. La dialectique trahison / recréation, qui soutient le traducteur de Shakespeare joue nettement moins pour Ben Jonson, car la déperdition est moins fréquemment compensée par une plus grande facilité de compréhension.

Est-ce à dire qu'on ne peut espérer rendre Ben Jonson intelligible que dans des éditions universitaires truffées de notes et qu'il faut donc renoncer à proposer un texte jouable qui ne soit pas une adaptation? Je ne le crois pas : les grandes comédies offrent un texte éminemment théâtral qui, même s'il ne fonctionne pas selon les mêmes principes que le texte shakespearien, pose aussi souvent des problèmes de traduction similaires, qui peuvent trouver des solutions semblables. 
Il faut rendre le comique verbal, fondé sur le vocabulaire (slogans, clichés, fausse science), et aussi sur quantité de jeux de mots ; ceux-ci sont souvent gratuits, parfois mauvais (comme celui sur «casa» - le mot espagnol - et «cozen», dans la scène où Subtle et Face croient berner un Espagnol qui n'est autre que Surly déguisé) ou dans le dialogue de sourds (au sens propre) entre Mosca et Corbaccio :

Corbaccio. How does your Patron?

Mosca. Troth as he did, Sir, no amends. Corbaccio.

What? Mendes he ?

(Volpone, I.1.6-7)

La plupart des jeux de mots sont incontournables parce qu'ils sont filés : ainsi on ne peut se dispenser de traduire les noms-étiquettes car la plupart donneront lieu à toutes sortes de calembours au cours de la pièce. Ainsi pour traduire le nom d'Abel Drugger, l'épicier, il faut prendre en compte la scène où Subtle lui concocte une enseigne sous forme de rébus :

He shall have a bel, that's Abel ;

And by it standing one whose name is Dee, In a rug gown, there's $\mathrm{D}$, and Rug, that's drug :

And right anenst him a dog snarling er ;

There's Drugger, Abel Drugger. That's his sign.

On ne peut donc traduire la liste des personnages avant d'avoir recensé les jeux de mots qui seront faits sur leurs noms : ma traduction de «Abel Drugger» par «Michel Lépicier» permet de jouer sur «l'épi scié», le prénom offrant le diminutif «Mimi» pour traduire «Nab»; l'allusion au célèbre alchimiste John Dee est nécessairement perdue.

Lorsque le pédantisme est exploité à des fins comiques (comme dans le discours de Nano mentionné plus tôt), on peut rendre l'érudition cocasse en traduisant les tribulations de l'âme de Pythagore en vers de mirliton.

Autre difficulté : le comique fondé sur les allitérations et souvent compliqué par des sous-entendus sexuels, comme dans la liste de promesses faites par Surly au faux Espagnol :

You shall be soaked, and stroked and tubb'd, and rubb'd, And scrubb'd, and fubb'd, dear don, before you go. 
You shall in faith, my scurvy baboon don,

Be curried, claw'd and flaw'd, and taw'd, indeed.

(The Alchimist, IV.3.96-100)

Ici comme ailleurs, il s'agit de trouver une langue orale non archaïsante qui rende tous les paramètres comiques perceptibles à nos contemporains, une langue aussi qu'un acteur puisse se mettre en bouche.

Car il est essentiel de retrouver le rythme comique, souvent fondé sur la rapidité des échanges, qui est l'un des atouts des grandes comédies de Jonson: ainsi, dans Volpone, les visites des divers clients (comme Corbaccio) sont caractérisées par la brièveté des répliques, les vers étant partagés entre les locuteurs; il importe alors de préserver ce rythme nerveux, tout en tenant compte de la respiration des acteurs. La traduction de ces comédies en vers exige la recherche constante de la concision, puisque notre équipe de traducteurs s'est donnée pour règle de ne pas dépasser 14 syllabes par vers, même pour les vers partagés; or, l'allongement est souvent inévitable lorsque l'on passe de l'anglais au français (sauf dans quelques cas très rares, comme lorsque le jeu du tutoiement et du vouvoiement, possible en français, permet de distinguer dans les discours échangés par Subtle et Face entre la complicité et la rivalité, et donc d'éliminer les nombreux «Sir» qui signalent la montée de la tension entre eux). Ce qui accroît la difficulté, c'est que Ben Jonson pratique volontiers l'accumulation, l'amoncellement, plutôt que le balancement, plus caractéristique du style shakespearien. Il arrive qu'un discours de Mammon (personnage dont la prétention est signalée par un style hyperbolique et une syntaxe aventureuse) soit constitué d'une seule phrase de 15 vers. Il faut alors s'efforcer d'en restituer le souffle. L'un des plaisirs de traduire se situe dans cette recherche d'un style, d'un ton, d'un rythme spécifiques à chaque personnage.

Il m'apparaît donc qu'on peut, qu'on doit, traduire Ben Jonson pour la scène et qu'il faut tenter de produire, dans la perspective de la représentation, un texte fidèle et vivant, qui pourra être oralisé, même s'il ne sera sans doute jouable que moyennant d'abondantes coupures; mais n'est-ce pas le lot de la plupart des pièces élisabéthaines mises en scènes ? J'ai eu la chance que ma traduction de Volpone donne lieu, en 1998, à une lecture publique, mise en espace par le Théâtre des deux rives de Rouen, et j'ai pu constater à cette occasion que les nombreux spectateurs présents n'avaient pas besoin du filtre de Jules Romains pour apprécier la pièce de Ben Jonson. 
Jean-Michel DÉPRATS. Il me reste à vous remercier tous les cinq de nous avoir éclairés et de nous avoir fait part de vos questionnements et de vos propositions de façon si limpide et si enrichissante.

Table ronde présentée par Jean-Michel DÉPR A TS Université Paris X - Nanterre

\section{NOTES}

'Shakespeare et la France, Société Française Shakespeare, Actes du congrès de 2000, textes réunis et présentés par Patricia Dorval, publiés sous la direction de Jean-Marie Maguin, p. 275-93. 